\title{
Research of the Integrated Methods of Multilevel Workflow and Multilevel BOM Configuration
}

\author{
Juan Jiang, Weiyan Xv and Xiufang Bai \\ Qingdao Binhai University, Qingdao, China
}

Keywords: Product data management; Multilevel workflow; Multilevel BOM configuration

\begin{abstract}
This paper proposes an integrated the technique of multilevel workflow and multilevel BOM configuration for the intractable problem on multilevel workflow and multilevel BOM configuration. The technique mainly refers to creating a mode of multilevel workflow and multilevel BOM configuration in PDM, a cooperative running model of workflow serves for BOM configuration and could effectively and harmoniously integrates multilevel workflow with multilevel BOM configuration.
\end{abstract}

\section{Introduction}

Product data management (PDM) is terms of the information management techniques which aims at product development [1-3]. There is a popular belief that tow main lines of PDM information management are static product structure and dynamic product design process. The information organization and resource management are centered on product design, which is different from other information management system, such as MIS, MRP and project management. Followed theories above, parts files generated by the workflow are manually configured to the structure tree. Manually configuring product structure increases the error rate. It is necessary to develop new BOM structure configuration method which generated dynamically by the project workflow and thence more according with the product process [4-7].

In the past there is integration of workflow management and BOM configuration: PDM workflow is corresponding with project, one project progress can generate many BOM configuration structures. In the beginning the project leader cannot know the detailed structure of the whole BOM configuration. Hence one workflow cannot satisfy the needs of the project task allocation, and the sub-process is necessary.

By developing PDM system, this paper thoroughly researches the PDM integration technique, mainly expounds integration methods of BOM configuration and workflow management in PDM system.

\section{Collaborative Operation Mode of Multilevel Workflow and Multilevel BOM Management}

Workflow. Stationary templates of workflow are used in some PDM system[8]. For example, the workflow only includes design, checkup, standardization, processing examine and authorize, or omits some of these items, which is short of flexibility. This workflow is completely linear, which can't realize bifurcate tasks and overlap tasks.

Workflow management in this paper consists of extra-workflow management and PDMBOX built-in workflow management. PDM BOX is a management model in which the personal users receive tasks and carry out works. When the project starts, the project leader actives extra-workflow, assigns the first level tasks. Then the first level task-receivers enter PDMBOX. If there are massive works that need assignment, the first level receivers can start built-in workflow in PDMBOX and assign second level tasks. In the same manner, a project can allocate countless levels of tasks, then generate countless sub-process. Each level workflow can select workflow template or user-defined template when selecting initial system. User-defined template is shown as Fig. 1. There is no limit of node numbers, and it allows bifurcate tasks and overlap tasks. The user can submit when the tasks were done, if the user cannot finish the tasks by him, he can allocate tasks to the next level. 


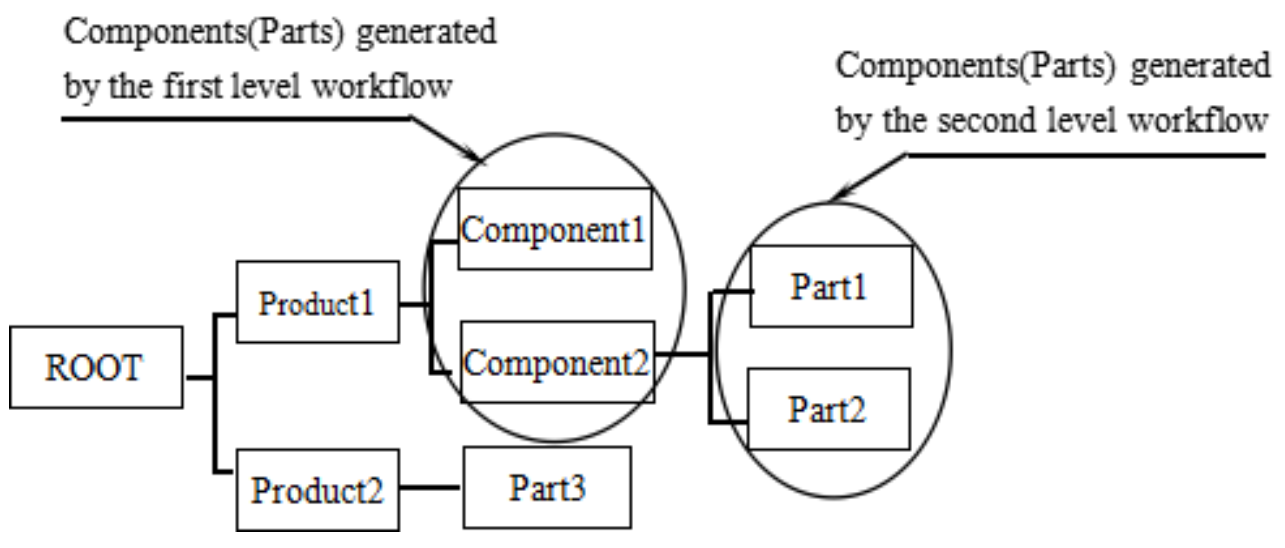

Figure 1. Sketch map of multilevel BOM expansion

This system supports concurrent workflows of multiple projects, also supports concurrent built-in workflows of different tasks in on project. It allows setting task relevance between workflow nodes. Task relevance means that the task of one user can be carried out or not be limited by another. For example, when attributing tasks in workflow, task of node B is subject to task of node A. User of node B cannot carry out its task if task of node A was not done.

It can initiate the workflow throughout the whole PDM process, such as in process of compile the specifications or in the process of design parts. The workflow becomes tools for product structure configuration and helps to manage the data of the enterprise.

Multilevel Workflow Management and Multilevel BOM Management Cooperation Model. The product structure configuration management system supports expansion of multi-project and multilevel BOM, which reflects assembly relationships of the parts, relates all engineering data and documents, It can hang up part papers generated by workflow automatically, and classify the versions, search the latest product data effectively, then realize safety and in integrity detection.

The product structure configuration BOM expands by traversing lists in the form of tree control or tree view. Product structure tree in Table 1 corresponding with Fig. 1. Each node in structure tree corresponds one list in table1.Each node's property and relative file are recorded in table 2. Product structure configuration includes add, delete and modify node property in product structural tree. The new added nodes can be classified as enterprise standard parts, purchased parts, basic parts and parts generated by workflow. Standard parts, purchased parts and basic parts can be added to the product structure tree by borrowed nodes.

Table 1 Relations of product components and parts

\begin{tabular}{|c|c|c|c|}
\hline Parent node & Child node & Mission & Project \\
\hline Root & Product 1 & nothing & Project 1 \\
\hline Root & Product 2 & nothing & Project 2 \\
\hline Product 1 & Component 1 & Task 1 & Project 1 \\
\hline Product 1 & Component 2 & Task 1 & Project 1 \\
\hline Product 2 & Component 3 & Task 2 & Project 2 \\
\hline Component 1 & Part 1 & Task 1-1 & Project 1 \\
\hline Component 1 & Part 2 & Task 1-1 & Project 1 \\
\hline Component 2 & Part 3 & Task 2-1 & Project 1 \\
\hline$\ldots$ & $\ldots$ & $\ldots$ & $\ldots$ \\
\hline
\end{tabular}

Table 2 Property of components and parts

\begin{tabular}{cccc}
\hline Part & Revision & Material & File \\
\hline Component 1 & 1 & A3 & $\ldots$ \\
Component 2 & 2 & Al & $\ldots$ \\
Part 1 & 1 & $\ldots$ & $\ldots$ \\
\hline
\end{tabular}

In initial project usually use product name as project name. Starting extra-workflow, named first 
level workflow. Accordingly a root-node that named with the project name is generated on product structure tree. First level project leader can add or modify the configuration on first level product structure tree, as shown in Fig .1. Component 2 comes from base parts or standard parts. First level leader borrows them from base part library and adds them to first level nodes without starting workflow. Component 1 is designed by the company, start first level workflow and distribute tasks to component 1 leader.

Component 1 leader enters PDMBOX and budgets, if need many peoples cooperation design component 1, starts internal-workflow named second level workflow. Leader of second level workflow enters PDMBOX, receives the tasks and carries out the works. Each level task configurations are recorded in Table 3. Parent-child relationships between each level workflow are recognized by field "Parent node". The second level workflow may generate one or more components (parts). The number of components (parts) is decided by the second level leader according to the need, which is no longer in the charge of the first level leader. The second level leader can use general template or user-defined dynamic template to assign the tasks. When second level workflow is done, part 1 and part 2 generated in this process will be connected to component 2 automatically. And so on, when task executor cannot accomplish the tasks independently in second level workflow, he can start the third level workflow.

Table 3 Task allocation

\begin{tabular}{ccccc}
\hline Task & Parent node & name & finish & project \\
\hline Component 1 & Product 1 & Second-level leader & Not done & Product 1 \\
Product 1 & root & First-level leader & Not done & Product 1 \\
\hline
\end{tabular}

UML is a mobile structured system [9-11]. Using UML model to show how multi-level workflow management and multilevel BOM management cooperating, shown as Fig .2. When the component leader can't accomplish the work in budget period and need to attribute the next level tasks, he can start the next level workflow. At the same time, the system will record the task distribution in Table 3 , and record BOM configuration in Table 1.

In conclusion, in this system the product structured tree is generated dynamically. There is different product structure tree in different period of extra-workflow. It can start next level workflow as required in workflow. The components generated by workflow will be connected to relative product structure tree automatically. Product con-structure configuration is graded responsibility. The first level leader doesn't limit the type of workflow template started by the second level leader, doesn't limit the member distribution, and doesn't limit the number of components. Each leader uses the components generated by each workflow to configure his product structure tree.

This shows that the integration project take workflow management as necessary tool for BOM configuration management, and centered on product design. 


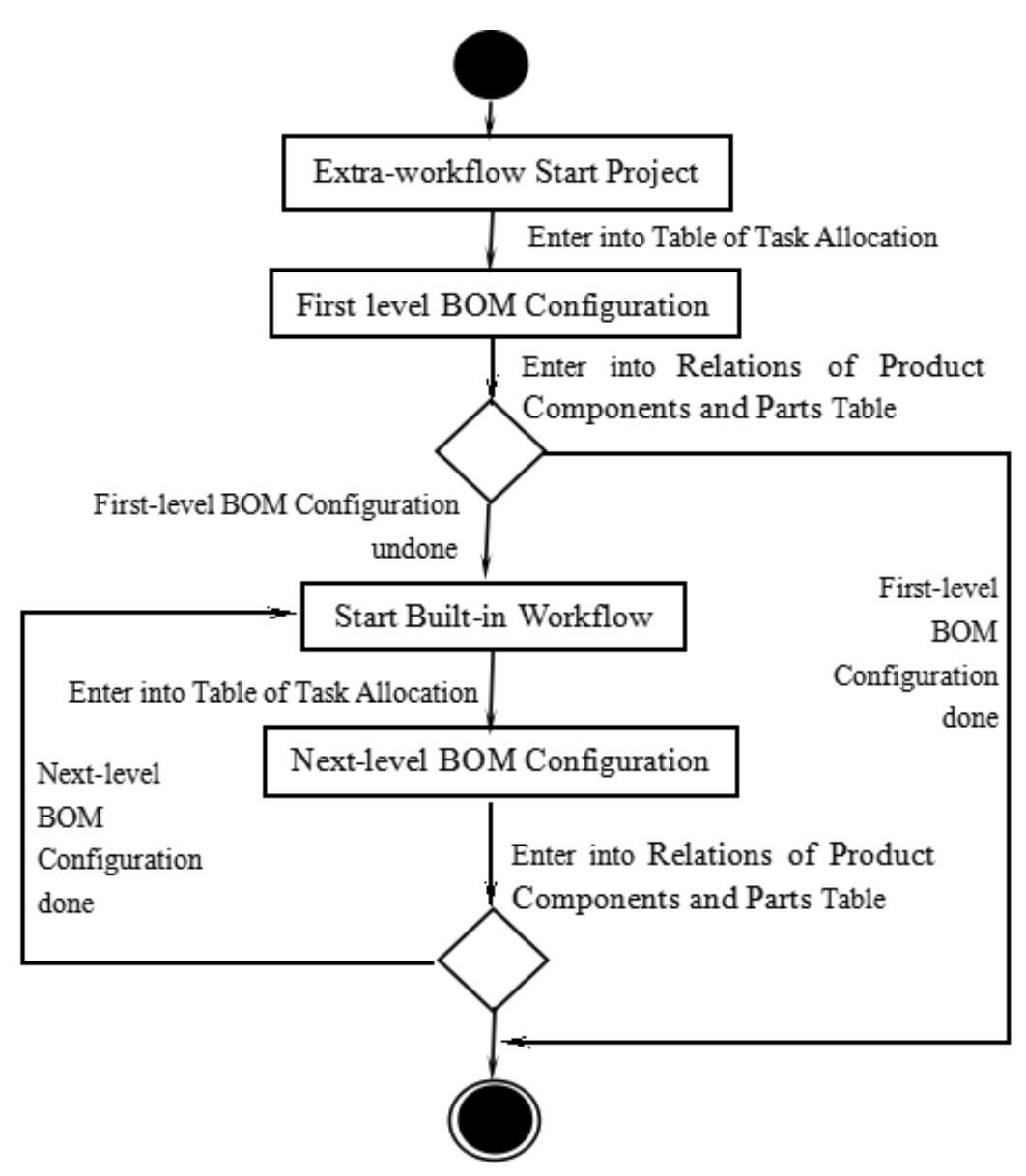

Figure 2. UML model of multilevel workflow management and multilevel BOM configuration cooperation

\section{Conclusion}

According to the integration thought, develop the PDM system, which based on C/S distribution web database system, using multi-user and multi-task concurrent cooperation, shown as Fig .2. Building the cooperation model can realize the cooperation of multilevel workflow management and multilevel BOM management in PDM. Provides a reliable reference for the in-depth study of PDM.

\section{References}

[1] X.F. Xia, L.L. Liang and J.J. Li: Journal of Chinese Computer Systems, Vol. 37 (2016) No.7, p.1440 (In Chinese).

[2] H.L. Jiang, Y.W. Liu and Q.Z. Fan: Computer Engineering and Design, Vol. 32 (2011) No.11, p.3682 (In Chinese).

[3] Tozawa Y and Yotsukura M: Integration of bills of material towards a communication tool[C]. Computer Science and Information Engineering, 2009 WRI World Congress on IEEE, 2009, 5: p446.

[4] L.J. He, L. Yi and L.C. Liu: Computer Engineering and Applications, Vol. 43(2007), No.15: p95 (In Chinese).

[5] X.W. Huang, Y.B. Song and X.B. Liu: Application Research of Computers, Vol. 26(2009), No.8: p2942 (In Chinese).

[6] K. Chen, S. Wei and C.A. Zhu: Computer Engineering, Vol. 34(2008), No.22: p34 (In Chinese). 
[7] Maltzahn S, Anderl R: ASME 2011 International Design Engineering Technical Conferences and Computers and Information in Engineering Conference, American Society of Mechanical Engineers, 2011: p1189.

[8] J.G. OU, Q.X. Chen and N. Mao: Die and Mould Industry, Vol. 35 (2009) No.9, p1 (In Chinese).

[9] W. Zhou, X.L. Zhang: Network Security Technology and Application, (2016) No.12, p59 (In Chinese).

[10]Benoit Eynard: Computers in Industry, Vol. 55,(2004):p301

[11]D. R. Shen, G. Yu and X. T. Wang: Journal of Software, Vol. 24(2013), No.8, p1786. 